\title{
The Gut Microbiome and Multiple Sclerosis
}

\author{
Javier Ochoa-Repáraz, ${ }^{1}$ Trevor O. Kirby, ${ }^{1}$ and Lloyd H. Kasper ${ }^{2}$ \\ ${ }^{1}$ Department of Biology, Eastern Washington University, Cheney, Washington 99004 \\ ${ }^{2}$ Department of Microbiology and Immunology, Geisel School of Medicine at Dartmouth College, \\ Lebanon, New Hampshire 03756 \\ Correspondence: jochoareparaz@ewu.edu
}

\begin{abstract}
The microbiome can be defined as the sum of the microbial and host's genome. Recent information regarding this complex organ suggests that in animal models of multiple sclerosis (MS), the composition of the gut microbiome can be altered, giving rise to both the effector and regulatory phases of central nervous system (CNS) demyelination. Experimental findings during the past decade in animal models of MS have provided clear evidence for the significant role of gut microbes in both the effector and regulatory phase of this condition. There is mounting evidence in preliminary human studies suggesting that a dysbiotic MS gut microbiome could affect disease progression. We propose considering the gut microbiome as a key organ for the regulation of tolerance mechanisms and speculate that the gut microbiome is the major environmental risk factor for CNS demyelinating disease. Accordingly, we hypothesize that intervention of the gut microbiome could result in safer novel therapeutic strategies to treat MS.
\end{abstract}

M ultiple sclerosis (MS) is a chronic autoimmune disease that affects the brain and spinal cord. Immune cells attack the myelin sheath that covers and protects neurons, resulting in a wide variety of neurological symptoms such as demyelination and axonal and neuronal loss (Dendrou et al. 2015). Most cases of relapsing MS are diagnosed in individuals between the ages of 20 and 50 with peak incidence at around 28. As noted in other autoimmune diseases, the gender ratio shows a higher frequency in females than in males. Some studies have shown evidence that suggests this ratio has shifted from 1:1, when MS was first described by Charcot at the end of the 19th century, to a current 3:1 female-to-male ratio (Fazekas et al. 2009). Fur- thermore, the gender ratio appears to be contingent on geographic location (Trojano et al. 2012). The etiological agent that may initiate disease remains unknown, and, although genetic susceptibility may be a factor, it cannot explain all instances (Compston and Coles 2008). The incomplete causative influence of genetic susceptibility suggests that other factors, such as environmental factors, might interact with the genetic susceptibility of the individual.

Whether MS is neurological or autoimmune by nature is still under debate (Dendrou et al. 2015), although most genetic profiling shows a preponderance of immune-mediated molecules that are activated during the disease process. Regardless of pathology, it is now understood

Editors: Howard L. Weiner and Vijay K. Kuchroo

Additional Perspectives on Multiple Sclerosis available at www.perspectivesinmedicine.org

Copyright (C) 2018 Cold Spring Harbor Laboratory Press; all rights reserved; doi: 10.1101/cshperspect.a029017

Cite this article as Cold Spring Harb Perspect Med 2018;8:a029017 
that inflammation and possibly immune dysregulation play a significant role during the progression of the disease. Both genetic and environmental factors have been proposed as putative factors that increase the risk for MS; some environmental risk factors include, but are not limited to, vitamin D deficiency, stress, smoking, viral or bacterial infections, and diet (Lincoln and Cook 2009; Mandia et al. 2014; Ochoa-Repáraz and Kasper 2014).

Within the last decade, a considerable effort has been made to ascertain a possible link between the functions of the human microbiome and human diseases. Public and private initiatives were launched to improve the technological tools needed for the study of the microbiome. It is possible now to evaluate the effects of genetic and environmental factors on the composition of the human microbiota as well as perform functional studies focused on the understanding of the metabolic or immunological impact of gut microbes. The gut microbiome is a bidirectional organ in which changes in the gut microbiota drive both immune and physiologic alterations in the host that in turn are critical in maintaining homeostasis of the gut microbiota. The current understanding of the complexity in the gut environment suggests the relevance of dysbiosis, which can be defined as alterations of the microbiome composition serving as a potential mechanism to cause disease. Dysbiosis can affect immunological responses to the microbiota, but further affect the integrity of the epithelia that comprise cellular barriers essential to maintaining the integrity of both intestine and central nervous system (CNS) (Fig. 1). A number of factors that have been associated with MS as potential triggers of disease appear to have significant effect on the gut microbial composition (Ochoa-Repáraz and Kasper 2014). Here, we review experimental evidence suggesting the vital importance of the gut microbiome in immune homeostasis as well as intestinal and blood-brain barrier (BBB) integrity. At present, there is both supporting and conflicting literature focused on the role of gut microbes in the regulation of MS and experimental autoimmune (or allergic) encephalomyelitis (EAE).

\section{THE GUT-ASSOCIATED LYMPHOID TISSUE IS A RESERVOIR FOR IMMUNE CELLS ASSOCIATED WITH MS}

Because of the deadly effects that pathogenic microbes have had in the history of human biology, we permanently link microbes with disease and illness. Nevertheless, among all gut microbes that have been identified thus far, only a minor fraction can be associated with disease. The rest of the microorganisms, including bacteria, archaea, fungi, eukaryotic microbial organisms, and viruses, are not harmful, and, in many cases, they are beneficial. Humans harbor an extremely large population of these commensal microbes throughout life, from birth to death. We globally name these microbes as the microbiota, and recognize the sum of the genes and products that are secreted or excreted by the microbiota as the microbiome. We now know that different locations of our bodies exposed to the environment around us comprise different microbiomes, including the skin, oral, respiratory tract, stomach, urogenital tract, and the gut. Furthermore, we are now beginning to understand how diverse these different microbiomes are and how different their functions might be. Of the microbiomes, the gut microbiome is the most studied because of its relative abundance and biological importance.

Anatomically, the gastrointestinal (GI) tract is a large muscular tube covered with a mucosal epithelium. The surface area of the human intestine is $\sim 200 \mathrm{~m}^{2}$. The gut of a human consists of the small intestine and the large intestine. Although the mucosal epithelium is present in both small and large intestines, there is variability in both morphology and function. The main function of the small intestine is to digest food and absorb nutrients, whereas the large intestine reabsorbs water and ions. The biological geography of the gut appears to affect the composition of the microbiota. Physical and chemical factors, such as the differences in morphology, enzymes, and acids released in the stomach as components of pancreatic juice and bile, and larger quantities of antimicrobial peptides released by Paneth cells, affect the microbial load of the small intestine, which is notably reduced when compared 
Gut Microbiome and Multiple Sclerosis

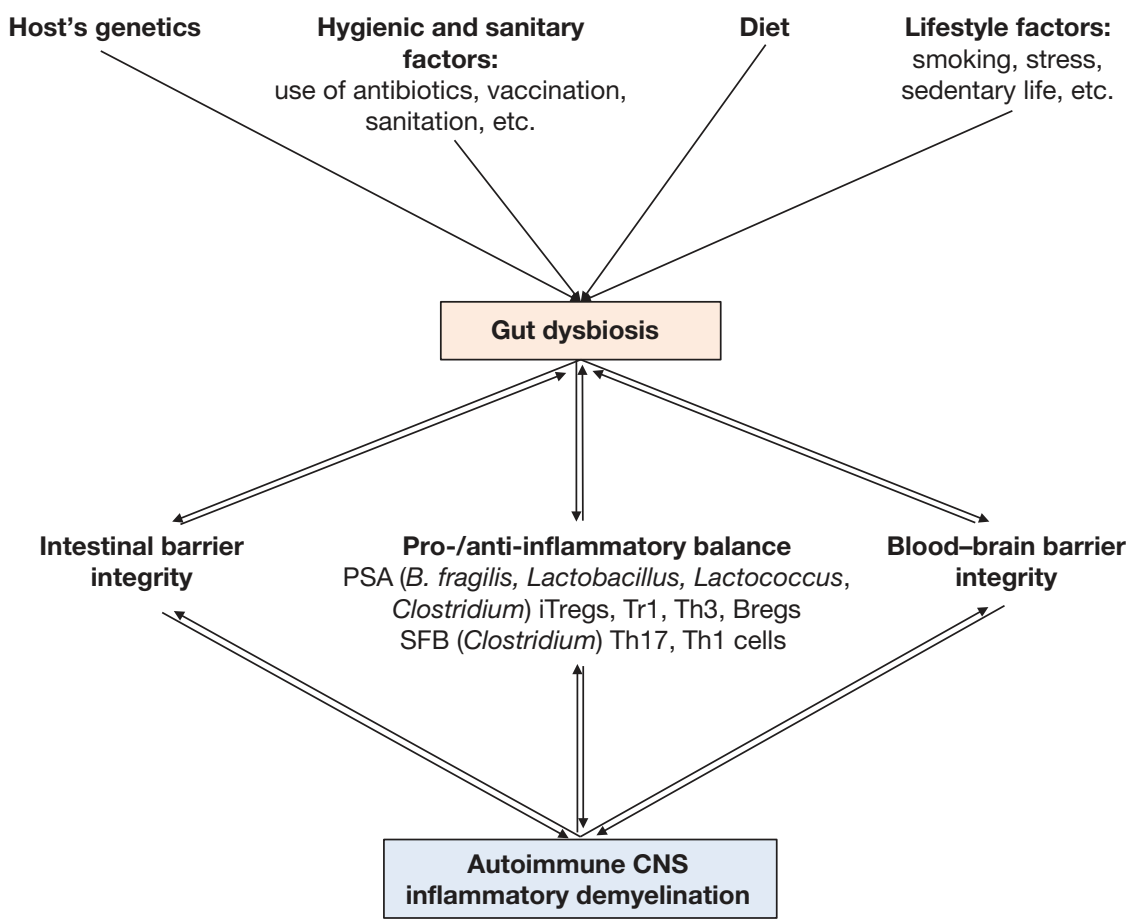

Figure 1. The reciprocal association of the gut dysbiosis with central nervous system (CNS) inflammatory demyelination. Host's genetics and distinct environmental factors, including diet, sanitation, use of antibiotics, and lifestyle, have been linked to increased multiple sclerosis (MS) risk. As reviewed by us, the same factors are known to affect the composition of the gut microbiota (Ochoa-Repáraz and Kasper 2014). Dietary factors that are believed to promote diverse proinflammatory pathways have been associated with gut dysbiosis, which is postulated as major factor for gut inflammatory diseases, but also hypothesized to be responsible for autoimmune CNS inflammatory diseases. Other dietary components promote anti-inflammatory responses that could help maintain a balanced gut microbiota. Both pro- and anti-inflammatory triggering dietary factors can have direct effects on the immune system. Also, metabolic products of microbial catabolism such as short-chain fatty acids (SCFAs) mediate in anti-inflammatory responses and intestinal barrier and blood-brain barrier integrity. Recent evidence suggests that the interaction between the gut microbiome and disease is bidirectional. Many aspects of the multifactorial interactions need to be elucidated using experimental models, but, more importantly, in human studies. PSA, Polysaccharide A; B. fragilis, Bacteroides fragilis; iTregs, inducible regulatory T cells; Bregs, regulatory B cells; Th, T helper; SFB, segmented filamentous bacterium.

with the large intestine. Other factors, such as the lower nutrient retention times as well as oxygen and $\mathrm{pH}$, also account for the longitudinal differences (Donaldson et al. 2015).

The gut microbiota is comprised of bacteria, archaea, viruses that infect bacteria (bacteriophages), fungi, and eukaryotic microbes. Of the microbiota, bacteria are most studied. The two most dominant phyla are Bacteroidetes and Firmicutes; these constitute some of the roughly 40 different species that the core gut microbiota is comprised of, resulting in $75 \%$ of the total abundance for at least 1 year in an individual (Martinez 2013). A second study argued that $60 \%$ of all bacterial species persisted for 5 years in individuals (Faith 2013). Taken together, it is evident that the gut microbiome is a highly complex ecosystem in which many factors merge and influence both the microbial communities and our immune, neuronal, and endocrine system (Ochoa-Repáraz and Kasper 2014). Because of its complexity, animal models are used to determine the biological relevance of gut microbes in the immune system. Gnotobiotic 
J. Ochoa-Repáraz et al.

(germ-free) mice, antibiotics, colonization with single or multiple microbial species, fecal transplantations, and also treatment with microbial antigens and metabolites are used to affect the microbiome and study the outcomes on immune populations.

Many of the observations that define the cellular and/or soluble mechanisms that contribute to MS development and progression are derived from experimental animal models, consistent with those currently being used to evaluate the effects of the gut microbiome on disease pathogenesis and regulation. The most widely used animal model is EAE. EAE is characterized by peripheral activation of $\mathrm{CD} 4^{+} \mathrm{T}$ helper 1 (Th1) and T helper 17 (Th17) cells in draining lymph nodes and infiltration of activated and proliferative Th1 and Th17 into the CNS by crossing the BBB from circulation. Both T-cell populations have been associated with immunological tolerance breakdown leading to autoimmunity. However, the sole involvement of Th1 cells in MS has been challenged with the identification of Th17 cells as drivers of CNS demyelination in EAE (Hofstetter et al. 2005; Komiyama et al. 2006; Sutton et al. 2006). Antibodies against interferon $\gamma$ (IFN- $\gamma$ ) (Billiau et al. 1988; Duong et al. 1994), IFN- $\gamma$ deficiency (Ferber et al. 1996; Chu et al. 2000), or IFN- $\gamma$ receptor deficiency in mice (Willenborg et al. 1996) exacerbate EAE severity. In contrast, T cells expressing interleukin (IL)-17 were found in cerebrospinal fluid and plaques of MS patients (Matusevicius et al. 1999), as well as an enhanced expression of IL-17 receptor (IL-17R) and IL-22 receptor (IL$22 \mathrm{R})$ in the vessels of MS lesions when compared to the vessels of healthy individuals (Kebir et al. 2007). In the EAE model, autoreactive $\mathrm{CD} 4^{+} \mathrm{T}$ cells cross the $\mathrm{BBB}$ and then are reactivated within the CNS by either resident tissuefixed macrophages or peripherally antigen-presenting cells (APCs) that may be either dendritic cells or possibly B cells.

Multiple efforts are now being taken to target another lymphoid cell population, B cells, with critical importance in the pathology of MS (Hauser 2015; Longbrake and Cross 2016). $B$ cells act both as an effector and as an APC and differentiate into plasma cells to produce anti- gen-specific antibodies. B cells recognize antigens with their B-cell receptor and mediate presentation in major histocompatibility complex (MHC) class II and costimulation. Major subsets of circulating B cells can produce proinflammatory cytokines, such as tumor necrosis factor $\alpha$ (TNF- $\alpha)$ and lymphotoxin. However, more restricted populations, such as B10 cells produce, anti-inflammatory cytokines such as IL-10 (Mauri and Menon 2015). B cells isolated from MS patients appear to be shifted toward the proinflammatory phenotype (BarOr et al. 2010). Furthermore, a recent published work has provided evidence to suggest that $\mathrm{B}$ cells from MS patients exacerbate neuronal antigen-specific Th17 cell responses in mechanisms mediated by B cells as APCs (Ireland et al. 2016).

In recent years, experimental evidence gathered from the rodent EAE models suggest that the gut microbiome, particularly changes in the gut microbiome, affect the frequencies or the function of many of the immune cells described above. Consequentially, the involvement of the gut microbiota in immune homeostasis and its association with autoimmune diseases, including MS, has been proposed. Although for decades the study of the gut microbes in the context of immune homeostasis was limited, it is now increasingly understood that the gut and the gut-associated lymphoid tissues (GALTs) are the reservoir for many of the lymphoid cells in our bodies. The hypothesis that at least part of the $10^{13}$ microbes that our gut harbors functionally affects the local as well as the systemic immune balance is no longer just speculation. In the next section, we review different mechanisms by which gut microbes and, more specifically, changes in the gut microbial composition, affects the numbers or the function of immune cells that are linked to MS pathology.

\section{HOW CAN GUT MICROBES REGULATE MS?}

The presence of gut microbes is required for the immune system's appropriate development and maturation. These properties are highlighted by the dramatic morphological, cellular, and functional deficiencies observed in germ-free 
animals when compared with conventionally housed animals (Round and Mazmanian 2009b). The immune dysfunction of germ-free mice is characterized by an unbalanced equilibrium between pro- and anti-inflammatory immune cells present systemically and, particularly, in the gut. These characteristics can be restored by the colonization of the gut with commensal microbes such as Bacteroides fragilis (Mazmanian et al. 2005). Thus, it is logical to postulate that gut microbes affect the proper maturation of the immune system as well as its function.

\section{Immunomodulation}

EAE models of gut microbiome absence, alteration, or bacterial monocolonization provide substantial experimental evidence to suggest that the microbes of the gut influence immune homeostasis by affecting the balance of those immune responses elicited to promote inflammation and those directed to control the extent of the immune response. For instance, segmented filamentous bacterium (SFB), a Gram-positive resident of the rodent gut significantly affects the severity of EAE, among other experimental models of autoimmunity (Lee et al. 2011). The mechanism by which SFB appears to enhance disease severity appears to be mediated by the activation of gut-induced Th17 cells. The monocolonozation of germ-free mice with SFB renders EAE disease-resistant germ-free mice susceptible by the induction of Th17 cells (Lee et al. 2011). Similar results have been observed in the germ-free mice model that is susceptible to rheumatoid arthritis (RA) (Chappert 2014; $\mathrm{Wu}$ et al. 2016). Other commensal microbes such as Porphyromonas gingivalis, a constituent of the oral microbiome, increases glial activation and are shown to exacerbate proinflammation and EAE severity. The lipopolysaccharide (LPS) of $P$. gingivalis was shown to be recognized by Toll-like receptor 2 (TLR2) and promoted neuroinflammation (Shapira et al. 2002; Nichols et al. 2009). Other bacteria taxa, such as specific clusters of Clostridium spp., have also been shown to promote enhanced Th17 cell responses in the gut (Horai et al. 2015). Interestingly, recent findings that will be discussed later in this work associate a significant increase in the relative abundances of specific clusters of Clostridium in stool samples obtained from relapsing remitting MS (RRMS) patients when compared to those from healthy donors (Miyake et al. 2015). The experimental evidence suggests Th17 cell induction by gut microbes, indicating that perhaps dysbiosis could influence the pool of inflammatory cells with an autoreactive potential. No causative association has been found; yet, two recent published works suggest a functional impact of the MS gut microbiota determined by fecal transplantation studies using germ-free mice as recipients (Berer et al. 2017; Cekanaviciute et al. 2017). Germ-free mice show reduced susceptibility to the disease when compared to conventionally housed mice, as previously published (Berer et al. 2011; Lee et al. 2011). In Cekanaviciute et al. (2017), the fecal content from individual MS patients or healthy donors were transferred into recipient C57 germ-free mice. Mice that received MS fecal content suffered a significantly more severe form of EAE when compared with controls, whereas those that received fecal content from healthy individuals developed significant reduction in the EAE clinical scores when compared with controls. In Berer et al. (2017), transgenic mice that develop spontaneous EAE transferred with fecal samples of MS individuals had an increase in the incidence of disease when compared with recipient mice that were transferred with fecal content isolated from their homozygotic siblings, not diagnosed with MS, and considered in their study healthy. Both studies highlighted the importance of IL-10-producing $\mathrm{CD} 4{ }^{+} \mathrm{T}$ cells in the immunodulatory effects of the gut microbiota (Berer et al. 2017; Cekanaviciute et al. 2017).

The immune system has evolved to protect multicellular organisms from pathogens; therefore, the immune system must be able to distinguish between pathogenic signals and commensal signals. In autoimmune diseases, autoreactive cells target self-antigens and harm cells promoting tissue disruption, inflammation, and disease. The acquisition of the adaptive immune system by vertebrates also evolved in the generation of mechanisms to control the presence and effects 
J. Ochoa-Repáraz et al.

of autoreactive cells. Central tolerance, which occurs in the thymus for developing $\mathrm{T}$ cells and in the bone marrow for maturing $\mathrm{B}$ cells, is a major mechanism for clonal deletion of autoreactive $\mathrm{T}$ and $\mathrm{B}$ cells by the induction of apoptosis. The process of self-restriction in response to a high-affinity interaction between the T-cell receptor and B-cell receptor to exposed self-antigens in the thymus and the bone marrow, respectively, result in the destruction of the majority of T and B cells. This process ensures that only those $\mathrm{T}$ and $\mathrm{B}$ cells that do not respond to selfantigens will result in mature effector cells. The loss of central tolerance is caused by the escape of self-reactive cells that do not undergo clonal deletion. Similarly, peripheral tolerance mechanisms attempt to prevent the destructive effects of autoreactive cells. Anergy induced by nonresponsiveness and direct interaction with regulatory cell populations are proposed as the main mechanisms by which our immune system destroys these cells in the periphery. Among the cell compartments that play a protective role against autoimmunity, the regulatory $\mathrm{T}$ cells (Tregs) are the most studied.

The immune dysfunction in MS, characteristic of other autoimmune conditions, may be caused by the loss of immune homeostasis arising from varying patterns of microbial colonization. As the gut is the main reservoir of immune cells, immune homeostasis in both the GALT and the periphery may be dependent on other microbes, inducing the necessary antiinflammatory responses.

Tregs are activated $\mathrm{CD} 4^{+} \mathrm{T}$ cells with enhanced expression of the $\alpha$-chain of the IL-2 receptor (IL-2R) or CD25, and the master regulator Fork-head box 3 (Foxp3). Two main types of Tregs have been identified thus far: the naturally occurring Tregs (nTregs) that are generated in the thymus, and the inducible Tregs (iTregs), generated in the periphery (Josefowicz and Rudensky 2009). The nTregs were first identified as an immunosuppressive $\mathrm{T}$-cell subset derived from the thymus (Sakaguchi et al. 1982) and later shown to express high levels of CD25 (IL-2R $\alpha$ chain) and identified as immunosuppressive (Sakaguchi et al. 1995). Subsequently, the transcription factor Foxp3 was discovered in the early 2000s as a critical gene responsible for the tolerance induced by Tregs (Chatila et al. 2000; Bennett et al. 2001). Foxp3 expression is required for Treg differentiation and suppressive activity, as well as for their proliferation and survival (Josefowicz and Rudensky 2009). Other important cell subsets have regulatory functions, including the already discussed regulatory B cells. Tr 1 cells and Th3 cells have also been shown to be critical players in tolerance. Tr1 cells are IL-10-producing $\mathrm{CD}^{+} \mathrm{T}$ cells that do not express Foxp3 (Sundstedt et al. 2003). Th3 cells were described by Howard Weiner's laboratory as transforming growth factor $\beta$ (TGF- $\beta$ )-producing $\mathrm{CD} 4{ }^{+} \mathrm{CD} 25^{+}$ $\mathrm{T}$ cells (Weiner 2001; Faria and Weiner 2006). Furthermore, invariant natural killer T (iNKT) cells have shown regulatory phenotypes (Nowak and Stein-Streilein 2007).

Tregs suppress the proliferation of other cells, affect the function of dendritic cells, and promote immune homeostasis. Immunosuppressive effects of Tregs are variable and include either direct cell-to-cell contact, the production of anti-inflammatory cytokines such as TGF- $\beta$ and IL-10, or indirect by the modulation of APC function. Because of their importance in controlling the proliferation of immune cells, there has been great interest in understanding the role of Tregs in MS development and their potential protective function against MS, as well as many other autoimmune diseases (Perdigoto et al. 2015). There is some uncertainty as to whether the absolute number of Tregs is reduced in individuals with MS compared to healthy controls. However, insufficient suppressive function of Tregs in individuals with MS has been described. Early studies compared the in vitro function of Tregs by studying the suppressive capacity of Tregs isolated from 15 MS patients when cocultured with proliferating effector $\mathrm{CD} 4^{+} \mathrm{CD} 25^{-}$ $\mathrm{T}$ cells (Viglietta et al. 2004). In MS patients, Tregs had a significantly reduced ability to suppress effector T-cell proliferation and reduced production of IFN- $\gamma$. Reduced immunosuppression was later confirmed by Haas and colleagues (Haas et al. 2005). Huan and collaborators (2005) detected reduced Foxp3 protein as well as reduced messenger RNA (mRNA) FOXP3 expression levels in MS patients; however, al- 
though their study included RRMS, secondary progressive MS (SPMS), and primary progressive MS (PPMS) patients, all samples were pooled for their analysis. In contrast, Venken and colleagues (Venken et al. 2006) reported that the frequencies of circulating Tregs in RRMS and SPMS patients were not different from those detected in healthy donors. However, they documented a significant reduction in the ability of Tregs isolated from RRMS to suppress the proliferation of effector $\mathrm{CD}^{+}{ }^{+} \mathrm{T}$ cells and their production of IFN- $\gamma$ in vitro. Interestingly, no suppressive dysfunction was observed in Tregs isolated from circulating blood of SPMS patients. To date, the reasons why the regulatory effects of Tregs might not be compromised in SPMS patients remain unknown (Fletcher et al. 2010). The differences may rely on the more pronounced inflammatory activity in relapsing forms of MS that would require the assistance from Tregs; however, this is a hypothesis that has yet to be tested. The suppressive ability of Tregs was associated with the levels of FOXP3 mRNA expression (Venken et al. 2006). Feger and collaborators (Feger et al. 2007) compared the frequencies of nTregs in the cerebrospinal fluid (CSF) and circulating blood of MS patients. They showed that, in CSF of MS patients, the frequencies of nTregs were enhanced, but no differences were observed in circulation (Feger et al. 2007). In their studies, all MS patients, including RRMS, SPMS, and PPMS, were also pooled for analysis. As indicated earlier, the distinct function of Tregs in the different clinical types of MS has yet to be elucidated. It would be important to determine whether Tregs of MS patients respond differently to inflammation at different stages of the disease process. Gut microbes are known to promote iTregs and provide an essential mechanism for the establishment of peripheral tolerance. Conversely, we are now beginning to appreciate that disease may affect the composition of the microbiota within the microbiome. Future studies will determine whether RRMS, PPMS, and SPMS patients have an identifiable influence on the microbiome. It would be important to evaluate whether the effects of those changes within the microbiota are the result of the regulatory function of Tregs. Another experimental barrier to overcome is the current inability to effectively discern the phenotype of nTregs and iTregs. Sorting this out would enhance our understanding of the role of natural versus microbiome-promoted iTregs in the context of the different forms of MS. As we will discuss in the next section, Fletcher and colleagues (2009) showed that both frequencies and suppressive function of circulating Tregs expressing the ectoenzyme CD39 are reduced in RRMS patients when compared with healthy individuals.

The EAE models have provided evidence to suggest that specific gut microbes can affect EAE severity. Some of these finding suggest a role for Tregs in protective immunity promoted by gut bacteria. Treatment of Lewis rats with Bifidobacterium animalis (Ezendam et al. 2008) and mice with a mixture of Lactobacillus spp. (Lavasani et al. 2010) regulate the extent of CNS inflammation and clinical scores in EAE.

B. fragilis is a major constituent of the human gut microflora comprising between $1 \%-3 \%$ of the total bacteria. B. fragilis produces eight extracellular polysaccharides that are essential for their ability to colonize and survive within the gut (Mazmanian et al. 2005, 2008; Surana and Kasper 2012). One of these polysaccharides, the polysaccharide A (PSA), is a potent immunomodulatory symbiont factor that is recognized by APCs in a TLR2-dependent mechanism that potentiates $\mathrm{CD} 4^{+} \mathrm{T}$-cell activation and induction of regulatory IL-10-producing cells (Mazmanian et al. 2005; Wang et al. 2006; Round and Mazmanian 2009a; Ochoa-Repáraz et al. 2010b). PSA produced by $B$. fragilis is protective in models of experimental colitis (Mazmanian et al. 2008; Dasgupta et al. 2014) and asthma (Johnson et al. 2015). The induced IL10 production that PSA stimulates in T cells is a common feature in the protective mechanism of action, although the phenotype of the $\mathrm{CD} 4^{+}$ T-cell population appears to be disease specific (Johnson et al. 2015). In murine EAE, PSA is protective prophylactically and therapeutically by the induction of IL-10-producing Tregs (Ochoa-Repáraz et al. 2010b; Wang et al. $2014 \mathrm{~b})$. The protection that PSA confers is not observed in IL-10-deficient mice, suggesting 
J. Ochoa-Repáraz et al.

that the anti-inflammatory cytokine is key in the immunomodulatory effects induced by PSA. Similarly, TLR2-deficient mice are unresponsive to the polysaccharide (Wang et al. 2014b). The recognition of PSA is mediated by both conventional (Mazmanian et al. 2005; Wang et al. 2006) and plasmacytoid dendritic cells (Dasgupta et al. 2014). PSA is then presented to $\mathrm{CD} 4^{+} \mathrm{T}$ cell by MHC class II-mediated mechanisms (Cobb and Kasper 2008, 2005).

In EAE mice, PSA enhances a phenotypically distinct subpopulation characterized by the expression of an ectoenzyme, the ectonucleoside triphosphate diphosphohydrolase 1 (ENTPD1), also known as CD39, responsible for the catabolism of ATP into adenosine monophosphate (AMP) (CD39 ${ }^{+}$Tregs) (Wang et al. 2014b). In vitro, Fletcher's findings showed the dysfunctional nature of the CD $39^{+}$Tregs isolated from MS patients when Th17 proliferation and IL-17 production were significantly reduced when compared with Tregs isolated from healthy donors. Of particular interest is the ability of this population of Tregs from both healthy and MS patients to suppress IFN- $\gamma$ production. CD39 hydrolyzes ATP into $5^{\prime}$ AMP. AMP is then catabolized to adenosine by another ectoenzyme, CD73 (Antonioli et al. 2013). ATP is released to the extracellular media when cells lyse and through lysis-independent mechanisms by nucleotide-permeable channels and by mediation of lysosomes (Antonioli et al. 2013). During tissue damage, as occurs during inflammation, ATP is released to the media. The expression of CD39 in immune cells appears to be regulated by the inflammatory milieu through cytokines, hypoxic cells, and oxidative stress (Antonioli et al. 2013). Adenosine has anti-inflammatory effects on T cells, including Tregs, by binding to their adenosine receptors.

The acquisition of a CD39 phenotype independent of Foxp3 by Tregs promoted by the treatment with PSA enhanced the expression of regulatory markers measured by flow cytometry and the production of IL-10. Furthermore, the acquisition of the $\mathrm{CD} 39^{+}$phenotype by regulatory $\mathrm{CD}^{+}{ }^{+} \mathrm{T}$ cells was associated with the upregulation of several activation markers (PD-1, GITR, CTLA-4, and ICOS) when compared with CD $39^{-}$T cells. The CD $39^{+}$Foxp3-negative population showed enhanced expression of surface-tracking molecules such as chemokine receptor-5 (CCR5), CCR6, and CXC chemokine receptor-3 (CXCR3), as well as the adhesion molecules CD49b and CD29 (Wang et al. 2014a). Most notable was the failure to protect CD39-deficient EAE mice by oral administration of PSA (Wang et al. 2014b). CNS levels of IL-17 of EAE mice are reduced when mice are treated orally with PSA. The adoptive transfer of PSA-CD $39^{+} \mathrm{CD}^{+} \mathrm{T}$ cells into RagKO recipient EAE mice (which lack mature $\mathrm{B}$ and $\mathrm{T}$ cells) induced protection, as opposed to $\mathrm{CD}^{-} 9^{-} \mathrm{CD} 4$ T cells (Wang et al. 2014b). The immunomodulatory and protective role of CD $39^{+}$Tregs that are induced in response to oral treatments with attenuated vaccine delivery vectors have also been shown in murine collagen-induced arthritis (CIA) (Kochetkova et al. 2010, 2011, 2014; Maddaloni et al. 2015). Ex vivo flow cytometry evaluation of the CNS of EAE mice confirmed that those animals treated with PSA had increased frequencies and numbers of $\mathrm{CD}_{3} 9^{+}$ $\mathrm{CD}^{+} \mathrm{T}$ cells, both Foxp3-positive and Foxp3negative (Wang et al. 2014a). Taken together, the findings observed with PSA suggest that gut microbes and derived factors can promote a regulatory phenotype in $\mathrm{T}$ cells that augments their migratory and, perhaps, their protective effects against CNS inflammatory demyelination. Promoting a Treg phenotype that includes the expression of CD39 could be a novel strategy to promote immunoregulation in the gut, and also systemically in an autoimmune scenario. As such, fingolimod, an approved MS therapeutic that blocks lymphocyte egress from lymph nodes by antagonizing the sphyngosin-1 phosphate-1 (S1P1) receptor enhances the levels of CD39 mRNA in circulating blood of MS-treated patients, as well as CD39 ${ }^{+}$Tregs measured by flow cytometry (Muls et al. 2014). We have observed that alemtuzumab, a humanized antiCD52 monoclonal antibody (mAb), enhances the expression of Foxp3 and CD39 in the circulating $\mathrm{CD}^{+} \mathrm{T}$-cell population that recovers after treatment (Mielcarz et al. 2013, presented at ECTRIMS 2013). In EAE mice treated with a murine anti-CD52 $\mathrm{mAb}$, the effects observed 
are similar (Pant et al. 2015, presented at ECTRIMS 2015). Remarkably, the CD39-positive phenotype is also exacerbated in GALT Tregs of mice treated orally with teriflunomide (ECTRIMS 2015) (Kircher et al. 2015), an approved oral therapy against relapsing forms of MS. Teriflonomide's described mechanism of action (MOA) is the inhibition of the de novo synthesis of pyrimidine that stops the proliferation of autoreactive cells by the action targeted against the enzyme dihydroorotate dehydrogenase $(\mathrm{DHODH})$. We observed an increase in the frequencies of GALT CD39 ${ }^{+}$Foxp3-negative $\mathrm{CD}^{+} \mathrm{T}$ cells and Tregs in naïve mice treated orally with daily doses of teriflunomide. Adoptive transfer of this isolated Treg population reduces significantly the severity of disease in recipient EAE mice. Our preliminary findings suggest an additional immunomodulatory MOA for the therapeutic based on GALT $\mathrm{CD} 9^{+}$-producing T cells. Based on our preliminary observations, we hypothesize that gut microbiome alterations lead to the long-term efficacy of some therapies. It appears that induction of a GALT-derived $\mathrm{CD} 39^{+}$population of regulatory $\mathrm{T}$ cells may offer a common pathway by which some and perhaps all of the Food and Drug Administration (FDA)-approved therapies for treating MS invoke their protective capacity. Accordingly, we speculate that the induction and the amplification of $\mathrm{CD} 39^{+} \mathrm{T}$ regulatory cells, which are dysfunctional in their ability to control Th17 cell proliferation and IL17 production by Th17 cells in MS patients (Fletcher et al. 2009), may result in improved efficacy of therapies for treating relapsing MS.

Recent clinical observations on the effect of anti-CD20 indicate that B cells should be considered a primary target to treat MS (Hauser 2015; Longbrake and Cross 2016). The enhanced severity of EAE in B-cell-deficient mice (Wolf et al. 1996) implies, as discussed previously, the existence of anti-inflammatory subsets of B cells, particularly those that produce IL-10 (Matsushita et al. 2008; Crampton et al. 2010). IL-10-producing B regulatory cells protect IL-10-deficient mice against EAE (Fillatreau et al. 2002). We have considered the impact of the gut microbiome on the phenotype and function of B cells during disease. In the gut, B cells can play a tolerogenic role against colitis (Wang et al. 2015). In colitis-induced mice, the disease is exacerbated in the absence of B cells and the number of GALT Tregs are reduced in B-cell-deficient mice. Based on their findings, it is suggested that Tregs and B cells are part of a tolerogenic loop wherein B cells promote the induction of Tregs and Tregs promote the maturation of immunoglobulin A (IgA)-producing plasma cells. Remarkably, the adoptive transfer of B cells obtained from IL-10-deficient mice also protected mice against colitis (Wang et al. 2015), suggesting an IL-10-independent immunomodulatory role for B cells (Ray et al. 2012). We have described that B cells isolated from mesenteric lymph nodes from B6 mice treated orally with antibiotics that target the microbiome were protective when transferred into recipient EAE mice (Ochoa-Repáraz et al. 2010a). The transferred B cells expressed increased levels of Cd1d and CD5. Interestingly, treatment with antibiotics also affected the function of Tregs during EAE (Ochoa-Repáraz et al. 2009). The more recent findings (Wang et al. 2015) and our previous work suggest that both Tregs and B cells might have a cooperative function during autoimmune inflammation. The induction of a B regulatory population by the microbiome may represent another important novel target for the treatment of this CNS inflammatory condition.

From the data and experiments presented, it becomes clear that there is an intimate relationship established between the immune system and the microbiota of the human microbiome. This relationship is contingent on the presence of resident microbes as well as the modulations of the host immune system. In summation, it is the microbiome that is partially responsible for maintaining a healthy balance between pro- and anti-inflammatory responses that regulate and educate the host's immune system, particularly in instances of autoimmunity.

\section{Gut-Derived Metabolites and the Control of Blood-Brain Barrier Permeability}

Metabolically, the presence of the gut microbiota has beneficial implications to the host 
J. Ochoa-Repáraz et al.

gut. Gut microbes process fibers from diet and produce metabolites with multiple diverse molecular and cellular functions in the metabolism and immune system of the host. Fermenting bacteria, such as members of the genera Clostridium and Bacteroides, of the colon are associated with these dietary fiber fermentation processes. Short-chain fatty acids (SCFAs), such as acetate, propionate, and butyrate, are products of the bacterial fermentation of nondigestible carbohydrates that are derived from complex fibers; in the absence of a gut microbiota, fibers would traverse the GI tract unused. SCFAs also provide immunological benefits to the host by regulating gene expression in immune cells and shifting them toward a regulatory phenotype, decreasing oxidative stress and reinforcing the intestinal barrier as well as the BBB. The importance of gut-derived metabolites was recently highlighted in the IL-10 knockout model of spontaneous colitis. In mice that suffer from severe intestinal epithelium barrier disruption, the treatment with a fiber-rich diet significantly reduced the disruption. The beneficial effects of the diet were accompanied by increases in the levels of acetate, propionate, and butyrate in the gut, a reduced inflammatory cell infiltration into the lamina propria and enhanced frequencies of Tregs (Wang et al. 2016). Thus, gut microbes could affect key metabolites associated with gut intestinal integrity as well as promotion of immunomodulatory responses. We speculate that metabolites such as SCFA produced in the gut affect the host systemically, as evidenced by their effects on BBB integrity. Although SCFA transport to the brain has been reported (Conn et al. 1983; Macfabe 2012), the migratory and effects mechanisms to and within the CNS require further investigation (Michel and Prat 2016).

During EAE, significant changes in metabolite patterns are found at the onset and at the peak of the disease of rats. In the blood of SJL EAE mice, $\sim 15 \%$ of the metabolites detected (283) differed in their concentrations during disease (Mangalam et al. 2013). Some of the gut microbes that produce SCFA as a result of fibers fermentation, such as clusters of Clostridium, have been recently shown to be significantly reduced in fecal samples obtained from
MS patients when compared with healthy donors (Miyake et al. 2015). Additionally, lipid 654, a bacteria-derived lipodipeptide produced by Bacteroides spp., is diminished in the serum of MS patients (Farrokhi et al. 2013). Other metabolites that may be present in the gut microbiota might also be associated with pathways of Th17 cell differentiation, such as sterols that have been shown to be ligands for ROR $\gamma \mathrm{t}(\mathrm{Hu}$ et al. 2015), for instance, oxysterols (Soroosh et al. 2014).

The importance of SCFA in the induction of regulatory responses mediated by Tregs has been extensively reviewed in recent years (Kinoshita and Takeda 2015). Recent findings suggest that these gut-derived metabolites could be implicated in processes with essential importance in inflammatory diseases of the CNS. Remarkably, the gut microbiome might be a key regulator of BBB integrity (Braniste et al. 2014). Germ-free mice show an increase in BBB permeability when compared to conventional specific pathogen-free (SPF) mice. Molecularly, the reduced integrity of the BBB was associated with an altered expression of occludin and claudin-5, which is significantly increased in the epithelium of germ-free mice. When fecal content obtained from SPF mice was transferred to GF mice, the BBB integrity improved and enhanced the expression of the tight junction proteins. Further, when germ-free mice were monocolonized with SCFA-producing gut bacteria, the integrity of the $\mathrm{BBB}$ was also restored (Braniste et al. 2014). In light of the findings, it appears that the permeability of the $\mathrm{BBB}$ in germ-free mice is underdeveloped and "leaky." As in MS, the integrity of BBB integrity is compromised; this is apparent in other CNS diseases as well (de Vries et al. 2012).

\section{HOW ABOUT THE OTHER WAY AROUND- DOES DISEASE AFFECT THE GUT MICROBIOME?}

As noted previously in this review, the gut microbiome is bidirectional. Microbiota drive a host immune or physiologic response and, in turn, the host maintains gut homeostasis and prevents the development of dysbiosis. During EAE, an 
increase in the frequencies of Th1, Th17, and IL$17^{+} \gamma \delta \mathrm{T}$ cells are observed in the GALT of mice as early as 7 days postdisease induction (Nouri et al. 2014). APCs isolated from the GALT of EAE mice also produce enhanced quantities of proinflammatory cytokines. Furthermore, the integrity of the intestinal barrier appears to be disrupted (Nouri et al. 2014). The leaky gut observed in EAE mice is also observed in a murine model of autism spectrum disorders (ASDs) (Hsiao et al. 2013). In this model, mice show GI dysfunction, dysbiosis, and increased intestinal permeability. Importantly, the treatment with $B$. fragilis improves the integrity of the barrier (Hsiao et al. 2013). The absence of microbes in germ-free mice also affects the intestinal barrier integrity (Kozakova et al. 2015) restored by gut microbes. The colonization with a probiotic, consisting of Lactobacillus rhamnosus LOCK0900, L. rhamnosus LOCK0908, and Lactobacillus casei LOCK0919, improved the intestinal morphology and barrier integrity (Kozakova et al. 2015). Thus, the effects of dysbiosis on the intestinal integrity could affect significantly the levels of gut microbial components, such as endotoxin (Cani et al. 2008), and exacerbate disease.

The findings obtained in the EAE model suggest that disease can affect intestinal integrity. Can MS also induce dysbiosis? The advances in the microbiological and population analytical tools, such as $16 \mathrm{~S}$ rRNA and metagenomic approaches, are starting to provide evidence that dysbiosis can occur in those with MS. Recent findings support the hypothesis of dysbiosis in MS (Rumah et al. 2013; Cantarel et al. 2015; Miyake et al. 2015; Chen et al. 2016; Jangi et al. 2016; Tremlett et al. 2016a, 2016b; Berer et al. 2017; Cekanaviciute et al. 2017). The presence of Clostriduim perfringens (Rumah et al. 2013), significant alterations in clusters of Clostridia (Miyake et al. 2015), reductions in Bacteroidaceae, and increases in Ruminococcus (Cantarel et al. 2015), uneven microbiota structures (Chen et al. 2016; Tremlett et al. 2016a, 2016b), and increases in Akkermansia municipalis, among other bacterial and archaeal taxa (Jangi et al. 2016; Berer et al. 2017; Cekanaviciute et al. 2017), have been described in MS gut micro- biota when compared with healthy individuals. Using the EAE model that afflicts the nonobese diabetic (NOD) strain of mice, we studied whether different stages of the disease would affect the composition of the gut microbiota. In the NOD mice, induced EAE promotes an early mild peak of disease that is followed by a period of remission and a subsequent worsening stage, prolonged and progressive (Basso et al. 2008; Levy et al. 2010). Histologically, the NOD EAE mice have changes that are observed in human MS during secondary progressive disease, including a leaky BBB. Our preliminary data suggest a significant difference $(p<0.017)$ in the overall microbial relative abundances of the gut microbiome in healthy versus mice that developed severe EAE during early stages of disease, measured at day 14 post-EAE induction. However, the overall microbiota structure of mice that developed severe EAE was not different to control mice at chronic stages of disease (Colpitts et al. 2017).

\section{CONCLUDING REMARKS}

Here, we reviewed the experimental evidence associating disease with various EAE models of the gut microbiome, including germ-free mice, oral antibiotics, colonization with single and multiple strains of gut microbes, and oral treatment with products obtained from gut commensal symbionts. Together, these experimental findings show that alteration of the GALTs or the metabolic pathways triggered by gut microbes affects the severity of CNS inflammatory demyelination. The experimental nature of these findings limit their potential clinical relevance, and, although still speculative, the responses that gut microbes elicit in the host suggest that they form part of a master regulatory system of immune responses that could potentially impact inflammatory CNS demyelinating disease. Preliminary studies have shown that the gut microbiome appears to be altered in those with relapsing MS. Recent preliminary evidence suggests that during disease the gut microbiome is at least affected quantitatively. Current efforts will determine whether the differences observed in the gut microbiome are translated into a func- 
J. Ochoa-Repáraz et al.

tionally biological relevance. More importantly, in the coming years, we will determine whether these effects that are now observed in the gut microbial populations and their functions can be used as potential novel therapies against MS. If so, this would represent a major paradigm shift in both our understanding as well as the treatment of this devastating condition.

\section{COMPETING INTEREST STATEMENT}

J.O.-R. reports grants from Genzyme, personal remuneration from Symbiotix Biotherapies, while conducting the study. L.H.K. reports grants from Genzyme, Teva and Symbiotix Biotherapies and personal remuneration from TEVA Neuroscience and Genzyme that are outside the submitted work.

\section{ACKNOWLEDGMENTS}

The authors acknowledge the support of the National MS Society grant CA 1027A1/3, the National Institutes of Health grants R41 AI110170/AI/NIAID and R56 AI098282/AI/ NIAID, and a Sanofi-Genzyme Corporate award (Genzyme/IST Concept Award \#GF-201411153).

\section{REFERENCES}

Antonioli L, Pacher P, Vizi ES, Haskó G. 2013. CD39 and CD73 in immunity and inflammation. Trends Mol Med 19: $355-367$

Bar-Or A, Fawaz L, Fan B, Darlington PJ, Rieger A, Ghorayeb C, Calabresi PA, Waubant E, Hauser SL, Zhang J, et al. 2010. Abnormal B-cell cytokine responses a trigger of T-cell-mediated disease in MS? Ann Neurol 67: 452-461.

Basso AS, Frenkel D, Quintana FJ, Costa-Pinto FA, PetrovicStojkovic S, Puckett L, Monsonego A, Bar-Shir A, Engel Y, Gozin M, et al. 2008. Reversal of axonal loss and disability in a mouse model of progressive multiple sclerosis. J Clin Invest 118: 1532-1543.

Bennett CL, Christie J, Ramsdell F, Brunkow ME, Ferguson PJ, Whitesell L, Kelly TE, Saulsbury FT, Chance PF, Ochs HD. 2001. The immune dysregulation, polyendocrinopathy, enteropathy, X-linked syndrome (IPEX) is caused by mutations of FOXP3. Nat Genet 27: 20-21.

Berer K, Mues M, Koutrolos M, Rasbi ZA, Boziki M, Johner C, Wekerle H, Krishnamoorthy G. 2011. Commensal microbiota and myelin autoantigen cooperate to trigger autoimmune demyelination. Nature 479: 538-541.
Berer K, Gerdes LA, Cekanaviciute E, Jia X, Xiao L, Xia Z, Liu C, Klotz L, Stauffer U, Baranzini SE, et al. 2017. Gut microbiota from multiple sclerosis patients enables spontaneous autoimmune encephalomyelitis in mice. Proc Natl Acad Sci 114: 10719-10724.

Billiau A, Heremans H, Vandekerckhove F, Dijkmans R, Sobis H, Meulepas E, Carton H. 1988. Enhancement of experimental allergic encephalomyelitis in mice by antibodies against IFN- $\gamma$. J Immunol 140: 1506-1510.

Braniste V, Al-Asmakh M, Kowal C, Anuar F, Abbaspour A, Toth M, Korecka A, Bakocevic N, Ng LG, Kundu P, et al. 2014. The gut microbiota influences blood-brain barrier permeability in mice. Sci Trans Med 6: 263 ra 158.

Cani PD, Bibiloni R, Knauf C, Waget A, Neyrinck AM, Delzenne NM, Burcelin R. 2008. Changes in gut microbiota control metabolic endotoxemia-induced inflammation in high-fat diet-induced obesity and diabetes in mice. Diabetes 57: 1470-1481.

Cantarel BL, Waubant E, Chehoud C, Kuczynski J, DeSantis TZ, Warrington J, Venkatesan A, Fraser CM, Mowry EM. 2015. Gut microbiota in multiple sclerosis: Possible influence of immunomodulators. J Investig Med 63: 729-734.

Cekanaviciute E, Yoo BB, Runia TF, Debelius JW, Singh S, Nelson CA, Kanner R, Bencosmed Y, Lee YK, Hauser SL, et al. 2017. Gut bacteria from multiple sclerosis patients modulate human $\mathrm{T}$ cells and exacerbate symptoms in mouse models. Proc Natl Acad Sci 114: E8943.

Chappert P. 2014. Role of SFB in autoimmune arthritis: An example of regulation of autoreactive T cell sensitivity in the gut. Gut Microbes 5: 259-264.

Chatila TA, Blaeser F, Ho N, Lederman HM, Voulgaropoulos C, Helms C, Bowcock AM. 2000. JM2, encoding a fork head-related protein, is mutated in X-linked autoimmunity-allergic disregulation syndrome. J Clin Invest 106: R75-R81.

Chen J, Chia N, Kalari KR, Yao JZ, Novotna M, Soldan MM, Luckey DH, Marietta EV, Jeraldo PR, Chen X, et al. 2016. Multiple sclerosis patients have a distinct gut microbiota compared to healthy controls. Sci Rep 6: 28484.

Chu CQ, Wittmer S, Dalton DK. 2000. Failure to suppress the expansion of the activated CD4 $\mathrm{T}$ cell population in interferon $\gamma$-deficient mice leads to exacerbation of experimental autoimmune encephalomyelitis. J Exp Med 192: 123-128.

Cobb BA, Kasper DL. 2005. Zwitterionic capsular polysaccharides: The new MHCII-dependent antigens. Cell Microbiol 7: 1398-1403.

Cobb BA, Kasper DL. 2008. Characteristics of carbohydrate antigen binding to the presentation protein HLA-DR. Glycobiology 18: 707-718.

Colpitts SL, Kasper EJ, Keever A, Liljenberg C, Kirby T, Magori K, Kasper LH, Ochoa-Repáraz J. 2017. A bidirectional association between the gut microbiota and CNS disease in a biphasic murine model of multiple sclerosis. Gut Microbes 14: 1-13.

Compston A, Coles A. 2008. Multiple sclerosis. Lancet 372: 1502-1517.

Conn AR, Fell DI, Steele RD. 1983. Characterization of $\alpha$ keto acid transport across blood-brain barrier in rats. Am J Physiol 245: E253-E260. 
Crampton SP, Voynova E, Bolland S. 2010. Innate pathways to B-cell activation and tolerance. Ann NY Acad Sci 1183: 58-68.

Dasgupta S, Erturk-Hasdemir D, Ochoa-Repáraz J, Reinecker HC, Kasper DL. 2014. Plasmacytoid dendritic cells mediate anti-inflammatory responses to a gut commensal molecule via both innate and adaptive mechanisms. Cell Host Microbe 15: 413-423.

Dendrou CA, Fugger L, Friese MA. 2015. Immunopathology of multiple sclerosis. Nature 15: 545-558.

de Vries HE, Kooij G, Frenkel D, Georgopoulos S, Monsonego A, Janigro D. 2012. Inflammatory events at bloodbrain barrier in neuroinflammatory and neurodegenerative disorders: Implications for clinical disease. Epilepsia 53: 45-52.

Donaldson GP, Lee SM, Mazmanian SK. 2015. Gut biogeography of the bacterial microbiota. Nat Rev Microbiol 14: 20-32.

Duong TT, Finkelman FD, Singh B, Strejan GH. 1994. Effect of anti-interferon- $\gamma$ monoclonal antibody treatment on the development of experimental allergic encephalomyelitis in resistant mouse strains. J Neuroimmunol 53: 101107.

Ezendam J, de Klerk A, Gremmer ER, van Loveren H. 2008. Effects of Bifidobacterium animalis administered during lactation on allergic and autoimmune responses in rodents. Clin Exp Immunol 154: 424-431.

Faith JJ, Guruge JL, Charbonneau M, Subramanian S, Seedorf H, Goodman AL, Clemente JC, Knight R, Heath AC, Leibel RL, et al. 2013. The long-term stability of the human gut microbiota. Science 341: 1237439.

Faria AMC, Weiner HL. 2006. Oral tolerance: Therapeutic implications for autoimmune diseases. Clin Dev Immunol 13: $143-157$.

Farrokhi V, Nemati R, Nichols FC, Yao X, Anstadt E, Fujiwara M, Grady J, Wakefield D, Castro W, Donaldson J, et al. 2013. Bacterial lipodipeptide, lipid 654, is a microbiome-associated biomarker for multiple sclerosis. Clin Transl Immunol 2: e8.

Fazekas F, Enzinger C, Wallner-Blazek M, Ropele S, PlutaFuerst A, Fuchs S. 2009. Gender differences in MRI studies on multiple sclerosis. J Neurol Sci 286: 28-30.

Feger U, Luther C, Poeschel S, Melms A, Tolosa E, Wiendl H. 2007. Increased frequency of $\mathrm{CD} 4^{+} \mathrm{CD} 25^{+}$regulatory $\mathrm{T}$ cells in the cerebrospinal fluid but not in the blood of multiple sclerosis patients. Clin Exp Immunol 147: 412418.

Ferber IA, Brocke S, Taylor-Edwards C, Ridgway W, Dinisco C, Steinman L, Dalton D, Fathman CG. 1996. Mice with a disrupted IFN- $\gamma$ gene are susceptible to the induction of experimental autoimmune encephalomyelitis (EAE). J Immunol 156: 5-7.

Fillatreau S, Sweenie CH, McGeachy MJ, Gray D, Anderton SM. 2002. B cells regulate autoimmunity by provision of IL-10. Nat Immunol 3: 944-950.

Fletcher JM, Lonergan R, Costelloe L, Kinsella K, Moran B, O’Farrelly C, Tubridy N, Mills KHG. 2009. CD $39^{+} \mathrm{Foxp}^{+}$ regulatory $\mathrm{T}$ cells suppress pathogenic Th17 cells and are impaired in multiple sclerosis. J Immunol 183: 76027610.
Fletcher JM, Lalor SJ, Sweeney CM, Tubridy N, Mills KHG. 2010. T cells in multiple sclerosis and experimental autoimmune encephalomyelitis. Clin Exp Immunol 162: 1-11.

Haas J, Hug A, Viehöver A, Fritzsching B, Falk CS, Filser A, Vetter T, Milkova L, Korporal M, Fritz B, et al. 2005. Reduced suppressive effect of $\mathrm{CD} 4^{+} \mathrm{CD} 25^{\text {high }}$ regulatory $\mathrm{T}$ cells on the $\mathrm{T}$ cell immune response against myelin oligodendrocyte glycoprotein in patients with multiple sclerosis. Eur J Immunol 35: 3343-3352.

Hauser SL. 2015. The Charcot Lecture | Beating MS: A story of B cells, with twists and turns. Mult Scler 21: 8-21.

Hofstetter HH, Ibrahim SM, Koczan D, Kruse N, Weishaupt A, Toyka KV, Gold R. 2005. Therapeutic efficacy of IL-17 neutralization in murine experimental autoimmune encephalomyelitis. Cell Immunol 237: 123-130.

Horai R, Zárate-Bladés CR, Dillenburg-Pilla P, Chen J, Kielczewski JL, Silver PB, Jittayasothorn Y, Chan C-C, Yamane $\mathrm{H}$, Honda $\mathrm{K}$, et al. 2015. Microbiota-dependent activation of an autoreactive $\mathrm{T}$ cell receptor provokes autoimmunity in an immunologically privileged site. Immunity 43: 343-353.

Hsiao EY, McBride SW, Hsien S, Sharon G, Hyde ER, McCue T, Codelli JA, Chow J, Reisman SE, Petrosino JF, et al. 2013. Microbiota modulate behavioral and physiological abnormalities associated with neurodevelopmental disorders. Cell 155: 1451-1463.

Hu X, Wang Y, Hao LY, Liu X, Lesch CA, Sanchez BM, Wendling JM, Morgan RW, Aicher TD, Carter LL, et al. 2015. Sterol metabolism controls $\mathrm{T}_{\mathrm{H}} 17$ differentiation by generating endogenous ROR $\gamma$ agonists. Nat Chem Biol 11: 141-147.

Huan J, Culbertson N, Spencer L, Bartholomew R, Burrows GG, Chou YK, Bourdette D, Ziegler SF, Offner H, Vandenbark AA. 2005. Decreased FOXP3 levels in multiple sclerosis patients. J Neurosci Res 81: 45-52.

Ireland SJ, Guzman AA, Frohman EM, Monson NL. 2016. B cells from relapsing remitting multiple sclerosis patients support neuro-antigen-specific Th17 responses. J Neuroimmunol 291: 46-53.

Jangi S, Gandhi R, Cox LM, Li N, von Glehn F, Yan R, Patel B, Mazzola MA, Liu S, Glanz BL, et al. 2016. Alterations of the human gut microbiome in multiple sclerosis. Nat Commun 7: 12015.

Johnson JL, Jones MB, Cobb BA. 2015. Bacterial capsular polysaccharide prevents the onset of asthma through $\mathrm{T}$ cell activation. Glycobiology 25: 368-375.

Josefowicz SZ, Rudensky A. 2009. Control of regulatory T cell lineage commitment and maintenance. Immunity 30: 616-625.

Kebir H, Kreymborg K, Ifergan I, Dodelet-Devillers A, Cayrol R, Bernard M, Giuliani F, Arbour N, Becher B, Prat A. 2007. Human $\mathrm{T}_{\mathrm{H}} 17$ lymphocytes promote blood-brain barrier disruption and central nervous system inflammation. Nat Med 13: 1173-1175.

Kinoshita M, Takeda K. 2015. Microbial and dietary factors modulating intestinal regulatory $\mathrm{T}$ cell homeostasis. FEBS Lett 588: 1-6.

Kircher C, Kasper E, Telesford KM, et al. 2015. A novel mechanism of action for teriflunomide by the induction of immunosuppressive gut $\mathrm{CD} 39^{+} \mathrm{FoxP}^{+}$Tregs in mice. Mult Scler 21: 546. 
J. Ochoa-Repáraz et al.

Kochetkova I, Golden S, Holderness K, Callis G, Pascual DW. 2010. IL-35 stimulation of CD $39^{+}$regulatory T cells confers protection against collagen II-induced arthritis via the production of IL-10. J Immunol 184: 7144-7153.

Kochetkova I, Thornburg T, Callis G, Pascual DW. 2011. Segregated regulatory $\mathrm{CD} 39^{+} \mathrm{CD} 4^{+} \mathrm{T}$ cell function: TGF- $\beta$-producing Foxp $3^{-}$and IL-10-producing Foxp $3^{-}$ cells are interdependent for protection against collageninduced arthritis. J Immunol 187: 4654-4666.

Kochetkova I, Thornburg T, Callis G, Holderness K, Maddaloni M, Pascual DW. 2014. Oral Escherichia coli colonization factor antigen I fimbriae ameliorate arthritis via IL-35, not IL-27. J Immunol 192: 804-816.

Komiyama Y, Nakae S, Matsuki T, Nambu A, Ishigame H, Kakuta S, Sudo K, Iwakura Y. 2006. IL-17 plays an important role in the development of experimental autoimmune encephalomyelitis. J Immunol 177: 566-573.

Kozakova H, Schwarzer M, Tuckova L, Srutkova D, Czarnowska E, Rosiak I, Hudcovic T, Schabussova I, Hermanova P, Zakostelska Z, et al. 2015. Colonization of germfree mice with a mixture of three lactobacillus strains enhances the integrity of gut mucosa and ameliorates allergic sensitization. Cell Mol Immunol 13: 251-252.

Lavasani S, Dzhambazov B, Nouri M, Fåk F, Buske S, Molin G, Thorlacius H, Alenfall J, Jeppsson B, Weström B. 2010. A novel probiotic mixture exerts a therapeutic effect on experimental autoimmune encephalomyelitis mediated by IL-10 producing regulatory T cells. PLoS ONE 5: e9009-e9011.

Lee YK, Menezes JS, Umesaki Y, Mazmanian SK. 2011. Proinflammatory T-cell responses to gut microbiota promote experimental autoimmune encephalomyelitis. Proc Natl Acad Sci 108: 4615-4622.

Levy H, Assaf Y, Frenkel D. 2010. Characterization of brain lesions in a mouse model of progressive multiple sclerosis. Exp Neurol 226: 148-158.

Lincoln JA, Cook SD. 2009. An overview of gene-epigeneticenvironmental contributions to MS causation. J Neurol Sci 286: 54-57.

Longbrake EE, Cross AH. 2016. Effect of multiple sclerosis disease-modifying therapies on $\mathrm{B}$ cells and humoral immunity. JAMA Neurol 73: 219-225.

Macfabe DF. 2012. Short-chain fatty acid fermentation products of the gut microbiome: Implications in autism spectrum disorders. Microb Ecol Health Dis 23: 1.

Maddaloni M, Kochetkova I, Jun S, Callis G, Thornburg T, Pascual DW. 2015. Milk-based nutraceutical for treating autoimmune arthritis via the stimulation of IL-10- and TGF- $\beta$-producing $\mathrm{CD} 39^{+}$regulatory T cells. PLoS ONE 10: e 0117825 .

Mandia D, Ferraro O, Nosari G, Montomoli C, Zardini E, Bergamaschi R. 2014. Environmental factors and multiple sclerosis severity: A descriptive study. Int J Environ Res Public Health 11: 6417-6432.

Mangalam A, Poisson L, Nemutlu E, Datta I, Denic A, Dzeja P, Rodriguez M, Rattan R, Giri S. 2013. Profile of circulatory metabolites in a relapsing-remitting animal model of multiple sclerosis using global metabolomics. J Clin Cell Immunol doi: 10.4172/2155-9899.1000150.

Martínez I, Muller CE, Walter J. 2013. Long-term temporal analysis of the human fecal microbiota revealed a stable core of dominant bacterial species. PLoS ONE 8: e69621.
Matsushita T, Yanaba K, Bouaziz J-D, Fujimoto M, Tedder TF. 2008. Regulatory B cells inhibit EAE initiation in mice while other B cells promote disease progression. J Clin Invest 118: 3420-3430.

Matusevicius D, Kivisäkk P, He B, Kostulas N, Ozenci V, Fredrikson S, Link H. 1999. Interleukin-17 mRNA expression in blood and CSF mononuclear cells is augmented in multiple sclerosis. Mult Scler 5: 101-104.

Mauri C, Menon M. 2015. The expanding family of regulatory B cells. Int Immunol 27: 479-486.

Mazmanian SK, Liu CH, Tzianabos AO, Kasper DL. 2005. An immunomodulatory molecule of symbiotic bacteria directs maturation of the host immune system. Cell 122: 107-118.

Mazmanian SK, Round JL, Kasper DL. 2008. A microbial symbiosis factor prevents intestinal inflammatory disease. Nature 453: 620-625.

Michel L, Prat A. 2016. One more role for the gut: Microbiota and blood brain barrier. Ann Transl Med 4: 15 .

Mielcarz D, Bergeron A, DeLong J, Sears C, Grady R, Channon J, Kasper L. 2013. Anti-CD52 therapy induces significant changes in $\mathrm{T}$ and $\mathrm{B}$ cell subset frequency and cytokine expression by B cells in multiple sclerosis patients. Mult Scler 19 (Suppl): P542.

Miyake S, Kim S, Suda W, Oshima K, Nakamura M, Matsuoka T, Chihara N, Tomita A, Sato W, Kim SW, et al. 2015. Dysbiosis in the gut microbiota of patients with multiple sclerosis, with a striking depletion of species belonging to Clostridia XIVa and IV clusters. PLoS ONE 10: 0137429 .

Muls N, Dang HA, Sindic CJM, van Pesch V. 2014. Fingolimod increases CD39-expressing regulatory $\mathrm{T}$ cells in multiple sclerosis patients. PLOS ONE 9: e113025.

Nichols FC, Housley WJ, O'Conor CA, Manning T, Wu S, Clark RB. 2009. Unique lipids from a common human bacterium represent a new class of Toll-like receptor 2 ligands capable of enhancing autoimmunity. Am J Pathol 175: $2430-2438$.

Nouri M, Bredberg A, Weström B, Lavasani S. 2014. Intestinal barrier dysfunction develops at the onset of experimental autoimmune encephalomyelitis, and can be induced by adoptive transfer of auto-reactive T cells. PLoS ONE 9: e106335.

Nowak M, Stein-Streilein J. 2007. Invariant NKT cells and tolerance. Int Rev Immunol 26: 95-119.

Ochoa-Repáraz J, Kasper LH. 2014. Gut microbiome and the risk factors in central nervous system autoimmunity. FEBS Lett 588: 4214-4222.

Ochoa-Repáraz J, Mielcarz DW, Ditrio LE, Burroughs AR, Foureau DM, Haque-Begum S, Kasper LH. 2009. Role of gut commensal microflora in the development of experimental autoimmune encephalomyelitis. J Immunol 183: 6041-6050.

Ochoa-Repáraz J, Mielcarz DW, Haque-Begum S, Kasper LH. 2010a. Induction of a regulatory B cell population in experimental allergic encephalomyelitis by alteration of the gut commensal microflora. Gut Microbes 1: 103108.

Ochoa-Repáraz J, Mielcarz DW, Wang Y, Begum-Haque S, Dasgupta S, Kasper DL, Kasper LH. 2010b. A polysaccharide from the human commensal Bacteroides fragilis pro- 
tects against CNS demyelinating disease. Muc Immunol 3: 487-495.

Pant A, Wang Y, Mielcarz DW, Mishra M, Channon JY, Telesford KM, Kasper EJ, Kircher C, Ochoa-Repáraz J, Haque A, et al. 2015. Anti-mu CD52 treatment induces $\mathrm{CD} 39^{+}$regulatory cells in the GALT and suppresses inflammatory cytokine expression within the CNS of EAE mice. Mult Scler 21: P539.

Perdigoto AL, Chatenoud L, Bluestone JA, Herold KC. 2015. Inducing and administering Tregs to treat human disease. Front Immunol 6: 654

Ray A, Basu S, Williams CB, Salzman NH, Dittel BN. 2012. A novel IL-10-independent regulatory role for $\mathrm{B}$ cells in suppressing autoimmunity by maintenance of regulatory T cells via GITR ligand. J Immunol 188: 3188-3198.

Round JL, Mazmanian SK. 2009a. Inducible Foxp $3^{+}$regulatory T-cell development by a commensal bacterium of the intestinal microbiota. Proc Natl Acad Sci 107: $12204-$ 12209.

Round JL, Mazmanian SK. 2009b. The gut microbiota shapes intestinal immune responses during health and disease. Nat Rev Immunol 9: 313-323.

Rumah KR, Linden J, Fischetti VA, Vartanian T. 2013. Isolation of Clostridium perfringens type B in an individual at clinical presentation of multiple sclerosis provides clues for environmental triggers of the disease. PLOS ONE 8: e76359.

Sakaguchi S, Takahashi T, Nishizuka Y. 1982. Study on cellular events in postthymectomy autoimmune oophoritis in mice. I: Requirement of Lyt-1 effector cells for oocytes damage after adoptive transfer. J Exp Med 156: 1565 1576.

Sakaguchi S, Sakaguchi N, Asano M, Itoh M, Toda M. 1995. Immunologic self-tolerance maintained by activated $\mathrm{T}$ cells expressing IL-2 receptor $\alpha$-chains (CD25). Breakdown of a single mechanism of self-tolerance causes various autoimmune diseases. J Immunol 155: 1151-1164.

Shapira L, Ayalon S, Brenner T. 2002. Effects of Porphyromonas gingivalis on the central nervous system: Activation of glial cells and exacerbation of experimental autoimmune encephalomyelitis. J Periodontol 73: 511-516.

Soroosh P, Wu J, Xue X, Song J, Sutton SW, Sablad M, Yu J, Nelen MI, Liu X, Castro G, et al. 2014. Oxysterols are agonist ligands of ROR $\gamma \mathrm{t}$ and drive Th17 cell differentiation. Proc Natl Acad Sci 111: 12163-12168.

Sundstedt A, O'Neill EJ, Nicolson KS, Wraith DC. 2003. Role for IL-10 in suppression mediated by peptide-induced regulatory T cells in vivo. J Immunol 170: 1240-1248.

Surana NK, Kasper DL. 2012. The yin yang of bacterial polysaccharides: Lessons learned from B. fragilis PSA. Immunol Rev 245: 13-26.

Sutton C, Brereton C, Keogh B, Mills KHG, Lavelle EC. 2006. A crucial role for interleukin (IL)-1 in the induction of IL17-producing $\mathrm{T}$ cells that mediate autoimmune encephalomyelitis. J Exp Med 203: 1685-1691.

Tremlett H, Fadrosh DW, Faruqi AA, Hart J, Roalstad S, Graves JO, Spencer CM, Lynch SV, Zamvil SS, Waubant E, et al. 2016a. Associations between the gut microbiota and host immune markers in pediatric multiple sclerosis and controls. BMC Neurol 16: 182.
Tremlett H, Fadrosh DW, Faruqi AA, Zhu F, Hart J, Roalstad S, Graves J, Lynch S, Waubant E; US Network of Pediatric MS Centers. 2016b. Gut microbiota in early pediatric multiple sclerosis: A case-control study. Eur J Neurol 23: 1308-1321.

Trojano M, Lucchese G, Graziano G, Taylor BV, Simpson S, Lepore V, Grand'maison F, Duquette P, Izquierdo G, Grammond P, et al. 2012. Geographical variations in sex ratio trends over time in multiple sclerosis. PLoS ONE 7: e48078.

Venken K, Hellings N, Hensen K, Rummens JL, Medaer R, D'hooghe MB, Dubois B, Raus J, Stinissen P. 2006. Secondary progressive in contrast to relapsing-remitting multiple sclerosis patients show a normal $\mathrm{CD} 4^{+} \mathrm{CD} 25^{+}$ regulatory T-cell function and FOXP3 expression. J Neurosci Res 83: 1432-1446.

Viglietta V, Baecher-Allan C, Weiner HL, Hafler DA. 2004. Loss of functional suppression by $\mathrm{CD} 4^{+} \mathrm{CD} 25^{+}$regulatory $\mathrm{T}$ cells in patients with multiple sclerosis. J Exp Med 199: 971-979.

Wang Q, McLoughlin RM, Cobb BA, Charrel-Dennis M, Zaleski KJ, Golenbock D, Tzianabos AO, Kasper DL. 2006. A bacterial carbohydrate links innate and adaptive responses through Toll-like receptor 2. J Exp Med 203: 2853-2863.

Wang Y, Begum-Haque S, Telesford KM, Ochoa-Repáraz J, Christy M, Kasper EJ, Kasper DL, Robson SC, Kasper LH. 2014a. A commensal bacterial product elicits and modulates migratory capacity of $\mathrm{CD} 39^{+} \mathrm{CD} 4 \mathrm{~T}$ regulatory subsets in the suppression of neuroinflammation. Gut Microbes 5: 552-561.

Wang Y, Telesford KM, Ochoa-Repáraz J, Haque-Begum S, Christy M, Kasper EJ, Wang L, Wu Y, Robson SC, Kasper DL, et al. 2014b. An intestinal commensal symbiosis factor controls neuroinflammation via TLR2-mediated CD39 signalling. Nat Commun 5: 4432.

Wang L, Ray A, Jiang X, Wang J-Y, Basu S, Liu X, Qian T, He R, Dittel BN, Chu Y. 2015. T regulatory cells and B cells cooperate to form a regulatory loop that maintains gut homeostasis and suppresses dextran sulfate sodium-induced colitis. Muc Immunol 8: 1297-1312.

Wang H, Shi P, Zuo L, Dong J, Zhao J, Liu Q, Zhu W. 2016. Dietary non-digestible polysaccharides ameliorate intestinal epithelial barrier dysfunction in IL-10 knockout mice. J Crohns Colitis 10: 1076-1086.

Weiner HL. 2001. Oral tolerance: Immune mechanisms and the generation of Th3-type TGF- $\beta$-secreting regulatory cells. Microbes Infect 3: 947-954.

Willenborg DO, Fordham S, Bernard CC, Cowden WB, Ramshaw IA. 1996. IFN- $\gamma$ plays a critical down-regulatory role in the induction and effector phase of myelin oligodendrocyte glycoprotein-induced autoimmune encephalomyelitis. J Immunol 157: 3223-3227.

Wolf SD, Dittel BN, Hardardottir F, Janeway CA. 1996. Experimental autoimmune encephalomyelitis induction in genetically B cell-deficient mice. J Exp Med 184: 22712278.

Wu HJ, Ivanov II, Darce J, Hattori K, Shima T, Umesaki Y, Littman DR, Benoist C, Mathis D. 2016. Gut-residing segmented filamentous bacteria drive autoimmune arthritis via T helper 17 cells. Immunity 32: 1-13. 


\section{$\&_{\mathrm{CSH}}^{\infty} \&$ Cold Spring Harbor

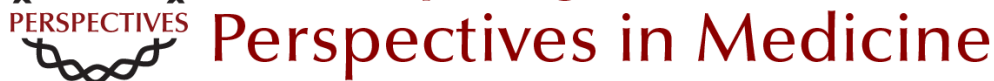

\section{The Gut Microbiome and Multiple Sclerosis}

Javier Ochoa-Repáraz, Trevor O. Kirby and Lloyd H. Kasper

Cold Spring Harb Perspect Med 2018; doi: 10.1101/cshperspect.a029017 originally published online January 8, 2018

\section{Subject Collection Multiple Sclerosis}

Multiple Sclerosis Pathology

Hans Lassmann

Regulatory T Cells: From Discovery to

Autoimmunity

Alexandra Kitz, Emily Singer and David Hafler

The Multiple Roles of B Cells in Multiple Sclerosis

and Their Implications in Multiple Sclerosis

Therapies

Rui Li and Amit Bar-Or

Autologous Hematopoietic Stem Cell

Transplantation in the Treatment of Multiple

Sclerosis

Carolina A. Rush, Harold L. Atkins and Mark S.

Freedman

B-Cell Therapies in Multiple Sclerosis

Joseph J. Sabatino, Jr., Scott S. Zamvil and Stephen L. Hauser

Oral Therapies for Multiple Sclerosis Simon Faissner and Ralf Gold

Interferon $\beta$ for Multiple Sclerosis

Dejan Jakimovski, Channa Kolb, Murali

Ramanathan, et al.

Alemtuzumab as Treatment for Multiple Sclerosis Serafeim Katsavos and Alasdair Coles

\author{
Natalizumab: Perspectives from the Bench to \\ Bedside \\ Afsaneh Shirani and Olaf Stüve \\ Daclizumab Therapy for Multiple Sclerosis \\ Bibiana Bielekova
}

Lifestyle and Environmental Factors in Multiple

Sclerosis

Lars Alfredsson and Tomas Olsson

Biomarkers in Multiple Sclerosis

Anu Paul, Manuel Comabella and Roopali Gandhi

The Evolving Mechanisms of Action of Glatiramer

Acetate

Thomas Prod'homme and Scott S. Zamvil

Regulation of Astrocyte Functions in Multiple Sclerosis

Michael A. Wheeler and Francisco J. Quintana

Experimental Autoimmune Encephalomyelitis

(EAE) as Animal Models of Multiple Sclerosis (MS)

Simon Glatigny and Estelle Bettelli

Neurodegeneration in Progressive Multiple

Sclerosis

Graham Campbell and Don Mahad

For additional articles in this collection, see http://perspectivesinmedicine.cshlp.org/cgi/collection/ 